\title{
A Simulation Approach to Evaluate the Effect of Demographic Changes on Healthcare Demand
}

Bozena Mielczarek ( $\sim$ bozena.mielczarek@pwr.edu.pl)

Wrocław University of Science and Technology

\section{Research article}

Keywords: Discrete event simulation, age-gender cohorts, healthcare demand

Posted Date: August 25th, 2020

DOI: https://doi.org/10.21203/rs.3.rs-62933/v1

License: @ (i) This work is licensed under a Creative Commons Attribution 4.0 International License. Read Full License 


\section{Abstract}

Background: The world's changing demographic trends inevitably lead to new challenges in the delivery of healthcare services. As the population ages, the consequent demand for various types of medical treatments changes as well, but the process is not uniform, neither for particular age-gender cohorts nor for different diagnoses' groups. Medium and long-term forecasts of future healthcare needs, which are crucial at the level of regional health policy decision making, should therefore be built taking into account different scenarios that describe possible alternative changes in population structure.

Methods: A discrete event simulation (DES) model was built to forecast future healthcare demand based on population projections for one Polish region. Demographic projections were formulated based on simulation experiments carried out in previous studies. Population forecasts were inputted into the DES model, together with patient data taken from the regional health fund. A simulation was run up to 2030 to predict future healthcare needs of older age-gender groups across different diagnosis categories. An additional experiment was performed with the hypothesis that admission rates for older cohorts will gradually increase starting from 2021.

Results: Three scenarios involved different assumptions regarding forecasted population changes as published by the Polish Government Council. The fourth scenario assumed gradually increasing admission rates expressed by the older age-gender groups. The results indicate that the expected small increase in the region's overall population will translate into a bigger increase in the number of patients. The largest increase of hospitalisations is projected for the age groups 75囚79 and older. The number of patients in the oldest women's group will be much higher than in the corresponding men's group. Meanwhile, the forecasted changes for particular disease categories are characterised by a high degree of variation, both in terms of gender and age.

Conclusions: A simulation provides wide possibilities for studying the impact of demographic trends in healthcare demand, as it allows conducting analyses in various cross sections. The results show that a change in the demographic structure and in particular, in the population's ageing, will lead to significant shifts in the distribution of health needs for hospital treatment.

\section{Background}

Demography is one of the main social aspects that need consideration in medium and long-term strategic economic planning. Governments in many developed countries are facing an emerging need to increase expenditures on pensions, health care, long-term care, and other areas directly related to demographics in an attempt to respond to significant changes in population structure. The main drivers of the observed demographic trends are the constantly increasing average life expectancy at birth and at retirement, and low birth rates that do not guarantee generational replacement. In Poland, the proportion of people aged above 65 years increased from 12.35\% in 2000 to $17.53 \%$ in 2018, and life expectancy increased from 78.00 years (women) and 69.74 (men) in 2000 to 81.68 (women) and 73.85 (men) in 2018.

Changes in the population structure inevitably lead to a rise in the number of older patients [1-3]. In addition, the development of new medical technologies, increasing knowledge on medical therapies, and easier access to medical care make the number of elderly patients grow even faster. The different effects (in varying degrees) of aging influence the demand for particular healthcare services, and these effects are strongly connected to the type of medical condition and the specific disease group [4,5]. Demographic ageing also has different impacts on the demand for healthcare services with respect to different levels of care, namely, primary care, secondary care, hospital day-care treatments, and hospitalisation [6]. Moreover, research on healthcare demand forecasting should be conducted differently for a single provider and at a whole region's level. In the latter case, an important issue to be considered when planning coverage of the population's needs is the structure of the population that inhabits smaller administrative districts. This structure may cause the demand to be unevenly distributed in a given area.

Health forecasting may be supported successfully by mathematical modelling [7]. Existing models can be classified according to different criteria. One of the most interesting classifications was proposed by Roberfroid et al. [8] who divided health forecasting models into: supply-based, demand-based, need-based, and benchmarking models. The authors have drawn attention to the importance of a comprehensive approach in long-term analysis and warn against focusing only on specific areas. Another classification relates to methodological aspects. There are two main basic approaches evidenced in the literature: analytic forecasting methods $[9,10]$ and simulation techniques. Soyiri and Reidpath [10] gave a brief overview of the available analytical forecasting techniques used to predict healthcare demand. Some interesting references may also be found in Astolfi et al. [7]. Analytic forecasting models are usually less demanding in terms of data requirement; however, their abilities to examine complex systems; relations between heterogeneous groups of objects; issues such as non-linearity, time delays, feedback loops, individual behaviour of patients; and many others are rather limited. Simulation models are capable of investigating the behaviour of complex systems, both at the micro- and macro-levels. A unique and powerful feature of simulation is the ease of carrying out analysis and testing of an unlimited number of less likely 'what-if' scenarios. A strong advantage of simulation is also the ease of mapping random phenomena that are inextricably linked to systems in which human factors play an important role.

There are numerous examples of the use of simulation methods in forecasting the demand for health services. Basic simulation methods are applied such as Monte Carlo (MC) [11], system dynamics (SD) [12], agent-based (ABS) [13], discrete event (DES) [14], and microsimulation (MSM) [15]. Some authors have proposed hybrid models by adding analytical approaches to the main simulation method [16] or by building a second supportive simulation model that uses another simulation technique [17].

In most cases, the respective study focuses on either one group of healthcare services or on one disease category. It is also a common practice to use external demographic forecasts. Population predictions are usually obtained from simulations performed using other models or downloaded from external databases. For example, Ansah et al. [18] projected demand for eye service using officially published demographic characteristics and population forecasts exported from another SD simulation model. The main limitation of the presented model was the assumption that there is a fixed proportional relationship between the number of residents aged $40+$ years with eye conditions and the number of public sector visits. An SD model with aging chains for diabetes was discussed in

Page $2 / 18$ 
Sugiyama et al. [12]. Estimates for the overall population were calculated based on available statistics. The authors have assumed constant values for diabetes incidence rates and dialysis initiation rates. A slightly different approach to address future demographic changes was proposed by Demir et al. [5]. The authors developed a DES model to evaluate the treatment pathways of patients with cataract. The forecasted level of demand was estimated based on historical patient data and adjusted for population growth rates. A similar approach was applied by Senese et al. [19] for Italy and Barber and Lopez-Valcarcel [20] for Spain. Both research groups elaborated an SD model and included regional population projections developed by the regional statistical office to simulate future demand for specialists at the regional level. The set of health histories was created by Davis et al. [21] who developed an MSM model to test policy options under demographic changes. Using a dynamic MSM model, an extensive research on forecasting the levels of dependency among the older population in England, was presented in Kingston et al. [15]. The authors created the base population using data from three longitudinal studies and then simulated the ageing of the population and the associated levels of dependency. The goal was not to estimate the demand for healthcare services but to study the dependency profiles of future older population. In the literature, we can also find a small group of studies in which forecasting future demand for health care takes into account, in addition to demographic trends, non-demographic factors. For example, Schofield and Earnest [22] have included in their analysis impact factors such as policy recommendations for shorter length of stay or the introduction of new technologies and treatments.

The brief overview above shows that health forecasting is carried out mainly for selected cohorts and for individual disease categories. Motivated by these observations we propose a DES model to investigate the impact of population ageing on the demand for inpatient hospital services in a region extending to the south-western part of Poland. In our model, we used demographic projections created for the region in question during our previous research [23,24]. As part of our earlier studies, we have developed an approach called 'hierarchical cohorting' based on an SD simulation paradigm. It uses historical demographic data to formulate forecasts for each age-gender group of a region's population. However, it should be emphasised that the DES model can also be populated with demographic projections developed under any other (e.g. analytical) approach or those derived from publicly available demographic projections. The DES model formulates health forecasts for different age-gender groups and various disease categories. Additionally, in one simulation experiment, we reject the assumption of constant values of incidence rates among the oldest population.

\section{Methods}

We developed a DES model based on a regional inpatient healthcare system to formulate hospital demand projections for one Polish administrative region. We decided to use the DES method because it enables the modelling of individual treatment histories and patients' stays in hospital wards. By using the DES method, we were able to define patient characteristics such as age, gender, home address, and clinical-specific attributes such as diagnosis, hospital ward, length of stay, basic medical consultations, and interventions. Another extremely important feature of DES is that it allows observing, over time, changes in the status of patients' health conditions and comparing the existing capacity of the healthcare system with the forecasted level of demand.

According to recently published surveys on the application of DES in the healthcare sector [25] the majority of published papers are unit-specific and focus on individual providers. This is primarily due to the need to track carefully the operational level processes of a healthcare facility. However, there are also reports [14] of the use of DES on a more aggregate level, especially when researchers intend to link global trends (e.g. demography) to treatment processes that relate to small, clearly distinguished patient groups. We designed the DES model using the Arena ${ }^{\circledR}$ software to examine the impact of demographic changes observed at the regional level on the intensity of admissions to hospital wards. Each simulation experiment consisted of 10 independent runs, each of which covered a full calendar year.

Simulation model

The simulation model focuses on the inflow of patients to 17 hospitals located in the Wrocław region (WR). The WR, which, together with three other regions, is a part of Lower Silesia-a big administrative province in southwest part of Poland-serves as Lower Silesia's main economic, scientific, and administrative centre. All hospitals are accessed by elective and emergency patients who mostly live in the region's capital or in any of the eight communes around it. Additionally, hospitals also admit patients from communes adjacent to the region and patients from other regions of Poland. The inhabitants of nine main communes (the capital plus eight adjacent municipalities) belong to 36 age-gender cohorts, which in turn generate 36 independent patient flows (Fig. 1). Affiliation to a particular cohort determines, based on the fitted random distribution, the commune of a patient's residence and the main diagnosis, which is then indicated on the hospital admission sheet (Fig. 2). The main diagnosis, in turn, is the premise for choosing the hospital to which the patient is referred. The hospital choice is thus strongly correlated with the age-gender cohort.

The next modelling phase describes patients' pathways within hospitals and allows mapping out the treatment and care received. However, this phase goes beyond the scope of this paper and will not be discussed here in detail.

Data collection and model parameters

The model is populated with data from two main sources. The medical and administrative details of all patients admitted in 2010 to WR hospitals were extracted from the branch registry of the Lower Silesian National Health Fund (NHF). In particular, two medical data sets were analysed. The first one describes 183,517 patients living in the WR who were registered in any hospital in Lower Silesia during 2010. The second data set encompasses 201,636 patients who were registered in one of the 17 hospitals located in the WR in 2010. The analysis of NHF datasets provided information regarding the spatial distribution of patients, arrival rates by calendar month, and patients' pathways during hospital treatment. The next set of source data included demographic projections formulated separately for 36 cohorts of the WR population through 2030. For this purpose we used the results of our earlier work [23,24] during which we simulated, among others, three main demographic scenarios (Table 1): the baseline scenario assumes a slight increase in life expectancy and a high increase in fertility; the high scenario assumes a high increase in life expectancy and a high increase in fertility; and the medium scenario assumes a small increase in life expectancy and a very high increase in fertility. 
For model validation, we used a data set that contains information on patients admitted to WR hospitals in 2011.

Fig. 1 Patients' arrival at Wrocław region (WR) hospitals. Legend: Each age-gender cohort of WR residents corresponds to patients who arrive at WR hospitals. Additionally, patients from Lower Silesia (LS) and other regions of Poland (PL) may also register at the WR hospitals.

Fig. 2 Relationships between patients' attributes in the discrete event simulation model

Table 1 The main scenarios for forecasting possible demographic changes in the Wrocław region population

\begin{tabular}{|ll|}
\hline Scenario & Description \\
\hline Scenario 1. Baseline scenario & High fertility rate values, medium mortality rate values, and migration unchanged \\
\hline Scenario 2. High scenario & High fertility rate values, low mortality rate values, and migration unchanged \\
\hline Scenario 3. Medium scenario & Very high fertility rate values, high mortality rate values, and migration unchanged \\
\hline
\end{tabular}

Model assumptions and output measures

Our study focuses on the number of patients who will be admitted to hospitals in the region over the next few years, broken down by age-gender cohorts and diagnosis groups. We look for the extent to which demographic changes (assuming stability of other factors) influence the incidence rates and affect the number of hospitalised patients.

The key assumption is based on the determination of age-gender specific demand rates. These values, interpreted as coefficients of use of hospital services, have been determined as the quotient of the number of hospitalisations performed on patients from a given age-gender subgroup and the population of the relevant cohort (Fig. 3). During the first phase of the study, it was assumed that the coefficients would remain stable for the entire forecast horizon. However, in the next experiment, this assumption was lifted, and the number of hospitalisations was examined taking into account increased values of demand rates for the oldest age groups.

Fig. 3 Age-gender specific demand rates for the Wrocław region population

The demand rates presented in Fig. 3 confirm differences in the number of hospitalisations, both in relation to gender and age. Higher rates can be observed among the male population in practically every age group, except for the two oldest cohorts: $75 \square 79$ and $85+$, for which higher rates are reported for the female population. For example, in the oldest female cohort (85+), for every 1,000 women there are 368 hospital visits per year, while in the male cohort ( $85+$ ), this rate is 347 visits per 1,000 inhabitants per year. Another distinct correlation characteristic of both genders are the lower values of the indicators for the population aged 20 to 50 years, slightly higher for the youngest age groups, and very high for the oldest cohorts.

In the DES model, the arrival of patients is based on the heterogeneous Poisson process. As demographic data are considered with a time step of one year, the simulation of the number of hospital visits is also determined at the end of each year. However, the data obtained from the NHF allow observing patient admissions on individual days, months, and quarters. Therefore, after a comprehensive analysis of the NHF data set we identified the month-of-the-year arrival patterns across every age-gender group and calculated the demand distribution parameters as the number of admissions per hour that is constant for the given month. This means that patients who arrive at the WR hospitals one at a time are independent of each other, and the number of arriving patients during a given calendar month is described by a Poisson random variable. The DES model works with data differentiated for each age-gender group. A characteristic feature of the arrival process is that more arrivals are observed in the summer, whereas fewer patients register in the winter.

Verification and validation of the model

We examined the validity of the model in several ways. Specifically, the model was validated using face validation, hypothesis testing, and historical validation [26]. The latter technique allows verifying the reliability of the model in relation to the correctness of the forecasts. For this purpose, we compared the results of the simulation for 2011 and the historical data obtained from the NHF regional branch. Table 2 shows mean absolute percentage error (MAPE) values when comparing the number of patients arriving at WR hospitals, grouped based on gender and by different ages. There is generally good agreement between the age-gender specific demand from the DES model and that from the NHF. The results of the simulation are consistent with historical data, although the model overestimates the oldest female cohorts slightly more strongly.

We also compared the annual distribution of patients arriving at the WR hospitals. The temporal distribution of patient arrivals exhibits, in some months, certain discrepancies, as compared with historical demand (Fig. 4). Larger differences can be observed in winter months; this is related to the significantly increased incidence of influenza. However, the averages calculated for each month for the validated year (2011) are highly convergent, indicating that the simulation model provides, on average, good results for the estimation of the WR demand.

Table 2 Validation of the simulation model: Annual number of patients registered in different age-gender groups 


\begin{tabular}{|llllllll|}
\hline & Historical & Simulation & MAPE & & Historical & Simulation & MAPE \\
\hline Female & 92284 & 91911.9 & $0.4 \%$ & Male & 92094 & 88850.2 & $3.5 \%$ \\
\hline F20+ & 71984 & 72991.8 & $1.4 \%$ & M20+ & 66655 & 65062.5 & $2.4 \%$ \\
\hline F30+ & 59500 & 62286.9 & $4.7 \%$ & M30+ & 52394 & 52722.5 & $0.6 \%$ \\
\hline F40+ & 48298 & 50934.1 & $5.5 \%$ & M40+ & 39672 & 39977.2 & $0.8 \%$ \\
\hline F50+ & 40774 & 43119.3 & $5.8 \%$ & M50+ & 30999 & 31372.0 & $1.2 \%$ \\
\hline F60+ & 28612 & 31974.9 & $11.8 \%$ & M60+ & 19172 & 20453.4 & $6.7 \%$ \\
\hline F70+ & 18796 & 21289.0 & $13.3 \%$ & M70+ & 10249 & 10953.1 & $6.9 \%$ \\
\hline F+M & 184378 & 180762.1 & $2.0 \%$ & & & & \\
\hline
\end{tabular}

Fig. 4 Monthly trends of arrivals by gender. Legend: A comparison of historical (2011) and simulation data (average values from 10 replications) for (a) female and (b) male population

We carried out a large number of tests to check the model's response to a sudden and rapid increase or decrease in demand. We also validated the model through visual demonstrations to NHF decision makers and healthcare professionals. After testing the model, we conclude that the model has a high degree of face validity.

\section{Results}

Demographic changes

All demographic scenarios forecast an increase in the WR population over the period 2018-2030, and the differences between scenarios are not significant. Extensive ageing of the population is predicted in every simulation scenario. According to the baseline scenario (Fig. 5), WR's population will grow from 1,225 thousand in 2018 to 1,272 thousand in 2030, and particularly strong growth is expected in the oldest age groups. The proportion of people aged above 65 years is likely to increase from its current level of 215 thousand in 2018 to 259 thousand in 2030 . Significant differences will persist between the population of the oldest women and men. In 2030, (Table 3) the population of women in the $65+$ cohorts will constitute from $23.22 \%$ (medium scenario) to $23.34 \%$ (high scenario) of the total population, while the population of men in the oldest cohorts will constitute from $17.08 \%$ (medium scenario) to $17.18 \%$ (high scenario) of the WR population. However, looking at the percentage increase in the oldest cohorts, we notice that between 2018 and 2030 the number of women in the $65+$ groups will increase by about $18.29 \%$ and for men, by $24.17 \%$ (baseline scenario).

Fig. 5 Demographic changes in the Wrocław region population according to the baseline scenario. Legend: the bar chart shows the projected population of the region from 2018 to 2030, and the solid line shows changes in the population aged 65+ years.

Table 3 Projected demographic changes in the WR population under three scenarios

\begin{tabular}{|c|c|c|c|c|c|c|c|c|c|c|c|c|}
\hline Year & Total pop & ation & & $65+$ coho & & & Women & & & Men 65+ & & \\
\hline Baseline & Medium & High & Baseline & Medium & High & Baseline & Medium & High & Baseline & Medium & High & \\
\hline 2018 & 1225411 & 1225076 & 1225076 & 214820 & 214810 & 214810 & 130763 & 130763 & 130763 & 84057 & 84047 & 84047 \\
\hline 2019 & 1234046 & 1233711 & 1233711 & 223242 & 223231 & 223231 & 135212 & 135212 & 135212 & 88030 & 88019 & 88019 \\
\hline 2020 & 1240339 & 1240280 & 1240020 & 231181 & 231170 & 231180 & 139587 & 139587 & 139593 & 91594 & 91582 & 91587 \\
\hline 2021 & 1246487 & 1246827 & 1246191 & 238545 & 238534 & 238561 & 143896 & 143896 & 143911 & 94649 & 94638 & 94651 \\
\hline 2022 & 1252100 & 1252823 & 1251821 & 244328 & 244317 & 244359 & 147046 & 147047 & 147070 & 97283 & 97271 & 97289 \\
\hline 2023 & 1257163 & 1258253 & 1256897 & 250102 & 250092 & 250149 & 150108 & 150109 & 150141 & 99993 & 99982 & 100008 \\
\hline 2024 & 1261467 & 1262924 & 1261225 & 255450 & 255441 & 255514 & 153011 & 153012 & 153051 & 102439 & 102429 & 102462 \\
\hline 2025 & 1265123 & 1266942 & 1264909 & 260535 & 260527 & 260613 & 155851 & 155853 & 155897 & 104684 & 104674 & 104716 \\
\hline 2026 & 1268739 & 1271329 & 1268581 & 264836 & 264830 & 264953 & 158403 & 158405 & 158470 & 106433 & 106426 & 106483 \\
\hline 2027 & 1267801 & 1271125 & 1267687 & 260848 & 260845 & 261005 & 155798 & 155800 & 155884 & 105050 & 105045 & 105121 \\
\hline 2028 & 1270334 & 1274372 & 1270257 & 261218 & 261218 & 261414 & 156067 & 156070 & 156173 & 105151 & 105147 & 105241 \\
\hline 2029 & 1270118 & 1274872 & 1270088 & 259065 & 259065 & 259295 & 154626 & 154629 & 154749 & 104439 & 104436 & 104547 \\
\hline 2030 & 1271806 & 1277275 & 1271826 & 259061 & 259061 & 259319 & 154684 & 154687 & 154821 & 104378 & 104374 & 104498 \\
\hline Change $\mathrm{e}^{\mathrm{a}}$ & $3.79 \%$ & $4.26 \%$ & $3.82 \%$ & $20.59 \%$ & $20.60 \%$ & $20.72 \%$ & $18.29 \%$ & $18.30 \%$ & $18.40 \%$ & $24.17 \%$ & $24.19 \%$ & $24.33 \%$ \\
\hline
\end{tabular}

${ }^{\text {a }}$ change between 2018 and 2030 
Overall demand for hospital services

The nominal values of regional demand for hospital services vary according to the age and gender of the patient. Fig. 6 shows the characteristic dependencies connected with the elapsing time of forecasts. The upward and downward trends in individual cohorts are clearly marked. For example, in cohorts $0 \otimes 5,20 \otimes 40$, and $60 \otimes 70$ the simulation forecasts a decrease in the number of patients, for both female and male. Meanwhile, in groups $5 \llbracket 20,40 \otimes 60$, and $70+$, a significant increase in the number of patients is predicted. Between 2018 and 2030, the highest percentage increase is expected for the 75-79 age group, at $53.4 \%$ and $67.4 \%$ for female and male cohorts, respectively (Fig. 7). In this and subsequent age groups, a significantly higher percentage increase is expected in male patients compared with female patients. However, in nominal values, the number of patients in the oldest group of women will be much higher than in the men's (Fig. 6).

Fig. 6 Total hospital demand according to the baseline scenario: Male population (left) and female population (right)

Fig. 7 Percentage changes in the total hospital demand between 2018 and 2030 according to the baseline scenario

Demand for hospital services by disease group and age-gender cohort

The incidence rates of individual diseases vary among different age-gender cohorts. This was the premise for the simulation of the prevalence by category of diseases, as classified according to the International Statistical Classification of Diseases and Related Health Problems (i.e. ICD-10), separately for each cohort. To facilitate the interpretation of results, the ICD codes were aggregated by the first letter (see Table 4) and in few cases by two letters or a letter and a number.

Table 4 Aggregated codes for IC10 disease classification

\begin{tabular}{|llll|}
\hline Descriptive name of the diesease category & ICD code & Aggregated code \\
\hline Certain infectious and parasitic diseases & A00 & B99 & AB \\
\hline Malignant Neoplasms & C00 & C97 & C \\
\hline In situ neoplasms & D00 & D48 & D1 \\
\hline Diseases of the blood & D50 & D89 & D2 \\
\hline Endocrine, nutritional and metabolic diseases & E00 & E90 & E \\
\hline Mental and behavioural disorders & F00 & F99 & F \\
\hline Diseases of the nervous system & G00 & G99 & G \\
\hline Diseases of the eye and adnexa. Diseases of the ear and mastoid process & H00 & H95 & H \\
\hline Diseases of the circulatory system & I00 & I99 & I \\
\hline Diseases of the respiratory system & J00 & J11 & J \\
\hline Diseases of the digestive system & K00 & K93 & K \\
\hline Diseases of the skin & L00 & L99 & L \\
\hline Diseases of the musculoskeletal system and connective tissue & M00 & M99 & M \\
\hline Diseases of the genitourinary system & N00 & N99 & N \\
\hline Pregnancy & O00 & Q99 & OQ \\
\hline Symptoms not elsewhere classified & R00 & R99 & R \\
\hline Injury, poisoning & S00 & T99 & ST \\
\hline
\end{tabular}

To determine future hospital demand trends between 2018 and 2030 for both gender populations and ICD-10 groups, compound annual growth rates (CAGR) were calculated for all ICD-10 groups listed in Table 4. Tables 5a and 5b present the CAGR values calculated according to formula (1):

$C A G R=\left(\frac{D_{2030}}{D_{2018}}\right)^{\left(\frac{1}{12}\right)}-1$

where and are the demand levels forecasted for 2018 and 2030, respectively, and 12 is the number of years from 2018 to 2030 . Empty cells in Tables 5 a and $5 \mathrm{~b}$ mean that the number of forecasted cases is so small that the calculation of CAGR values could be misleading. Cohorts with the highest decrease are marked in green, while those with the highest increase in demand are marked in red. It is evident that the predicted demographic changes have a very strong impact on morbidity trends, and as expected, demographic trends are echoed in the number of patients from individual cohorts.

Table 5a Compound annual growth rates (CAGR) [\%] calculated for demand (males) between 2018 and 2030 


\begin{tabular}{|c|c|c|c|c|c|c|c|c|c|c|c|c|c|c|c|c|c|c|}
\hline cohorts & $A B$ & C & D1 & D2 & $E$ & $\mathrm{~F}$ & G & $\mathrm{H}$ & I & $J$ & $\mathrm{~K}$ & L & $\mathrm{M}$ & $\mathrm{N}$ & $\mathrm{OQ}$ & $\mathrm{R}$ & ST & All \\
\hline $0-4$ & -0.58 & & & & & & -1.47 & -0.61 & & -0.66 & -0.49 & -0.60 & -0.67 & -0.69 & & -0.63 & -0.60 & $-0 . \epsilon$ \\
\hline $5-9$ & 0.30 & & & 0.22 & & & 0.19 & 0.32 & & 0.41 & 0.56 & 0.42 & 0.67 & -0.18 & & 0.24 & 0.41 & 0.3 \\
\hline $10-14$ & 1.18 & & & & & & 1.75 & 0.92 & & 1.05 & 0.08 & 2.01 & 1.63 & 0.92 & & 1.49 & 1.42 & 1.3 \\
\hline $15-19$ & 1.39 & & & & & & 1.80 & 1.35 & & 1.34 & 0.74 & 0.87 & 1.68 & 1.84 & & 1.30 & 1.48 & 1.4 \\
\hline $20-24$ & -0.57 & & & 0.72 & & 0.15 & 0.00 & -0.07 & 0.50 & 0.10 & 0.04 & -0.24 & -0.05 & 0.18 & & 0.08 & 0.12 & 0.0 \\
\hline $25-29$ & -0.49 & & & -0.49 & & -0.16 & -0.90 & -1.58 & -0.79 & -1.49 & -1.46 & -1.38 & -1.45 & -1.04 & & -1.15 & -1.34 & $-1 . \Xi$ \\
\hline $30-34$ & -2.08 & & & & & -1.95 & -2.64 & -2.76 & -2.51 & -2.92 & -2.71 & -2.44 & -2.13 & -1.95 & & -2.72 & -2.67 & $-2 . \epsilon$ \\
\hline $35-39$ & -2.25 & & & & -2.49 & -2.66 & -2.40 & -1.95 & -2.23 & -2.11 & -3.00 & -2.56 & -2.65 & -2.27 & & -2.45 & -2.30 & $-2 . \Xi$ \\
\hline $40-44$ & 0.08 & & & -1.60 & & -0.29 & 0.05 & -0.09 & -0.50 & -0.32 & -0.09 & -0.37 & -0.13 & -0.19 & & -0.41 & -0.18 & -0.2 \\
\hline $45-49$ & 1.17 & & & & & 2.84 & 1.99 & 1.99 & 1.89 & 2.10 & 2.54 & 2.27 & 2.60 & 2.33 & & 2.33 & 2.30 & 2.2 \\
\hline $50-54$ & 1.62 & 1.53 & 2.76 & & 3.88 & 2.34 & 3.13 & 2.78 & 3.33 & 3.14 & 3.45 & 3.16 & 2.46 & 3.02 & & 2.89 & 2.91 & 2.9 \\
\hline $55-59$ & 1.09 & 0.81 & 0.99 & & 0.74 & & 1.07 & 1.14 & 1.46 & 1.68 & 1.13 & 0.69 & 1.32 & 1.20 & & 1.17 & 1.26 & 1.2 \\
\hline $60-64$ & -1.81 & -1.22 & 0.14 & -1.12 & -1.31 & -0.78 & -0.64 & -0.68 & -0.66 & -0.32 & -0.80 & -1.14 & -0.44 & -0.70 & & -0.83 & -0.73 & $-0 . \overline{1}$ \\
\hline $65-69$ & -1.00 & -1.02 & -0.40 & & -0.92 & & -1.64 & -0.79 & -0.99 & -1.61 & -1.00 & -0.71 & -1.25 & -0.95 & & -1.03 & -1.01 & $-1 . c$ \\
\hline $70-74$ & 1.09 & 1.01 & 1.51 & & 1.64 & & 1.34 & 1.68 & 1.33 & 1.41 & 1.14 & 2.14 & 1.24 & 1.23 & & 1.41 & 1.26 & 1.3 \\
\hline $75-79$ & & 3.49 & 3.76 & 3.56 & 3.78 & & 4.12 & 3.76 & 3.96 & 4.04 & 4.74 & & 3.24 & 3.29 & & 4.04 & 4.13 & 3.9 \\
\hline $80-84$ & & 2.89 & 3.31 & & 3.65 & & 3.14 & 3.12 & 3.30 & 3.75 & 3.32 & & 3.87 & 3.17 & & 3.45 & 3.72 & 3.4 \\
\hline $85+$ & & 0.48 & & & 0.90 & & 1.50 & 1.38 & 0.96 & 1.28 & 1.59 & & 1.55 & 0.99 & & 1.03 & 1.43 & 1.1 \\
\hline All & -0.12 & 0.75 & $1.29^{\mathrm{c}}$ & 0.68 & 0.86 & 0.21 & 0.30 & 0.42 & 1.09 & 0.07 & 0.47 & 0.11 & 0.27 & 0.35 & 0.00 & 0.51 & 0.17 & 0.3 \\
\hline
\end{tabular}

All numbers are given as percentages and calculated according to the baseline scenario, by disease category and age-gender cohorts; ${ }^{a}$ Cohorts with the lowest CAGR values; ${ }^{b}$ Cohorts with the highest CAGR values; ${ }^{c}$ the highest CAGR value calculated for male population. Empty cells mean that the number of forecasted cases was too small to calculate valid CAGR values.

Table 5b Compound annual growth rates (CAGR) [\%] calculated for demand (females) between 2018 and 2030 


\begin{tabular}{|c|c|c|c|c|c|c|c|c|c|c|c|c|c|c|c|c|c|c|}
\hline cohorts & $A B$ & C & D1 & D2 & E & $\mathrm{F}$ & G & $\mathrm{H}$ & 1 & $\mathrm{~J}$ & K & L & $M$ & $\mathrm{~N}$ & $\mathrm{OQ}$ & $\mathrm{R}$ & ST & All \\
\hline $0-4$ & -0.67 & & & & & & & -0.55 & & -0.54 & -0.66 & -0.35 & -0.27 & -0.73 & & -0.69 & -0.54 & -0 \\
\hline $5-9$ & 0.30 & & & & & & 0.75 & 0.41 & & 0.32 & 0.75 & 0.97 & 0.93 & 0.91 & & 0.40 & 0.41 & 0.4 \\
\hline $10-14$ & 1.63 & & & & & & 0.87 & 1.63 & & 1.66 & 1.95 & 1.14 & 2.50 & & & 1.38 & 1.55 & $1 . !$ \\
\hline $15-19$ & 1.23 & & & & & & 2.03 & 1.33 & & 1.31 & 0.83 & 1.74 & 1.14 & 1.61 & 1.53 & 1.35 & 1.31 & 1.8 \\
\hline $20-24$ & -0.88 & & & & & -0.54 & -0.18 & -0.02 & 0.43 & 0.06 & -0.04 & 0.06 & 0.65 & -0.26 & 0.34 & -0.05 & 0.05 & 0.1 \\
\hline $25-29$ & -1.82 & & & & & & -0.83 & -1.39 & -1.75 & -1.25 & -1.50 & -1.78 & -1.10 & -0.93 & -1.60 & -1.38 & -1.24 & -1. \\
\hline $30-34$ & -2.70 & & & & & -2.95 & -2.67 & -2.53 & -2.62 & -2.96 & -3.00 & -2.24 & -2.47 & -2.81 & -2.49 & -2.71 & -2.55 & -2 \\
\hline $35-39$ & -2.16 & & & & -3.36 & -1.93 & -2.10 & -2.22 & -2.47 & -1.89 & -2.80 & -2.91 & -2.06 & -2.26 & -2.17 & -2.13 & -2.12 & -2 \\
\hline $40-44$ & 0.22 & & & & 0.11 & -0.02 & 0.35 & -0.24 & -0.07 & -0.24 & 0.30 & -0.21 & 0.16 & -0.49 & 1.02 & 0.25 & 0.05 & 0.1 \\
\hline $45-49$ & 3.60 & & & 3.40 & 1.87 & 1.31 & 2.85 & 2.45 & 2.44 & 2.60 & 2.93 & 2.98 & 2.69 & 2.42 & & 2.38 & 2.65 & $2 . !$ \\
\hline 50-54 & 1.60 & & & & 2.63 & 4.02 & 3.57 & 3.48 & 2.96 & 3.35 & 3.08 & 3.26 & 3.38 & 2.76 & & 3.23 & 3.23 & 3.: \\
\hline 55-59 & 0.98 & 1.52 & 0.37 & & 0.23 & 0.34 & 0.66 & 0.89 & 1.00 & 1.07 & 1.37 & 0.37 & 1.07 & 1.16 & & 0.88 & 0.99 & $0 . ؟$ \\
\hline $60-64$ & -1.68 & -0.44 & -0.61 & & -1.83 & -1.90 & -1.44 & -1.32 & -1.36 & -1.30 & -1.56 & -0.77 & -1.40 & -1.17 & & -1.46 & -1.23 & -1. \\
\hline $65-69$ & -1.59 & -1.64 & -2.10 & & -1.69 & & -1.59 & -1.31 & -1.44 & -1.53 & -1.19 & -1.92 & -1.01 & -1.96 & & -1.40 & -1.56 & -1. \\
\hline 70-74 & 0.90 & 0.66 & 1.26 & 1.38 & 1.16 & & 0.71 & 1.46 & 1.27 & 1.17 & 1.36 & 1.55 & 0.83 & 1.43 & & 1.15 & 1.06 & 1.2 \\
\hline $75-79$ & 4.69 & 3.52 & & 2.45 & 3.86 & 2.35 & 3.35 & 3.46 & 3.70 & 3.06 & 3.70 & 3.49 & 3.57 & 4.01 & & 3.27 & 3.45 & 3.4 \\
\hline $80-84$ & 2.36 & & & & 2.36 & & 2.36 & 2.30 & 2.34 & 2.09 & 2.07 & 3.33 & 2.68 & 2.43 & & 2.31 & 2.26 & 2. \\
\hline $85+$ & & & & 0.92 & 0.31 & & 0.12 & 0.24 & 0.39 & 0.03 & 0.12 & 0.73 & 0.44 & 0.37 & & 0.39 & 0.36 & 0.8 \\
\hline All & -0.05 & 0.73 & 0.71 & $1.02^{\mathrm{c}}$ & 0.25 & 0.13 & 0.40 & 0.47 & $1.04^{\mathrm{C}}$ & 0.06 & 0.41 & 0.33 & 0.66 & 0.10 & -1.52 & 0.45 & 0.45 & 0.4 \\
\hline
\end{tabular}

All numbers are given as percentages and calculated according to the baseline scenario, by disease category and age-gender cohorts; ${ }^{\text {a }}$ Cohorts with the lowest CAGR values; ${ }^{b}$ Cohorts with the highest CAGR values; ${ }^{c}$ the highest CAGR value calculated for male population. Empty cells mean that the number of forecasted cases was too small to calculate valid CAGR values.

Both the female and male populations will experience a significant increase in incidence rates in two age groups: $45 \rrbracket 54$ and $75 \llbracket 84$. However, comparing individual age groups in both populations, a higher growth is expected in the $50 \otimes 54$ female age group than in the corresponding male cohort. For the age group $80 \otimes 84$, there is an opposite upward trend: higher CAGR values are forecasted for the male cohort. The analysis of individual disease categories reveals very high CAGR values in D1 (men) and in D2 and I categories (women). This upward trend is primarily the result of a significant anticipated increase in the number of patients from the oldest cohorts.

Demand by selected disease code for older cohorts

For further analysis, the selection was limited to disease categories for which the proportion of patients aged 70 years and more in the total number of cases is the highest (Fig. 8).

The projections are different for each category of ICD, but there are some significant trends that result directly from demographic drivers. The higher proportion of women in the oldest age group is correlated in different ways with the number of predicted cases. In the categories of ICD-C (malignant neoplasms) and ICD-I (circulatory system) comparably fewer cases will be diagnosed in 2030 in the population of women than in men despite the fact that the population of women over 70 years of age (baseline scenario) is predicted to exceed the male population by 50 . Moreover, for the ICD-C category a more rapid increase in the male population is predicted, while in the female population the progression of this disease category is not so dynamic.

In the category ICD-E (metabolic diseases), there is a large rise in the incidence in the age group of women $65 \llbracket 69$ while in the male population, the increases are spread over several age groups. Furthermore, the overall growth rate is higher for all male cohorts. In addition, the overall growth rate is higher for all male cohorts, while in the female cohorts, a higher growth rate over the period 2018-2030 can only be observed in the oldest age group, 70+. In the case of nervous system diseases (ICD-G), the percentage increase of cases between 2018 and 2030 in the population as a whole is the smallest compared with other disease categories, but the increase in the oldest group is one of the highest, see Table 6.

Fig. 8 Forecasts of the incidence rates in selected disease groups. Legend: The graphs show those groups of diseases for which a significantly high share of patients aged over 70 years is observed

Table 6 Percentage increase of demand between 2018 and 2030 for selected disease categories 


\begin{tabular}{|lllllllll|}
\hline & All F & All M & M 65+ & M 65+ & F 70+ & M 70+ & F 75+ & M 75+ \\
\hline C & $10.6 \%$ & $16.9 \%$ & $22.1 \%$ & $22.4 \%$ & $32.4 \%$ & $36.7 \%$ & $32.1 \%$ & $43.3 \%$ \\
D1 & $11.1 \%$ & $17.7 \%$ & $19.5 \%$ & $27.0 \%$ & $34.6 \%$ & $41.4 \%$ & $36.8 \%$ & $47.3 \%$ \\
\hline E & $7.0 \%$ & $12.1 \%$ & $7.3 \%$ & $14.1 \%$ & $30.1 \%$ & $39.4 \%$ & $30.3 \%$ & $45.7 \%$ \\
\hline G & $4.9 \%$ & $3.8 \%$ & $15.1 \%$ & $25.2 \%$ & $23.7 \%$ & $43.3 \%$ & $27.4 \%$ & $50.9 \%$ \\
I & $15.0 \%$ & $15.1 \%$ & $25.1 \%$ & $27.7 \%$ & $28.5 \%$ & $39.2 \%$ & $30.1 \%$ & $45.4 \%$ \\
\hline
\end{tabular}

The effect of ageing on the occurrence of neurological diseases

Neurologic diseases are the major cause of death and disability in elderly patients [27]. They include mainly disorders of the brain, spine, and the nerves that connect them. Neurological diseases occur in all age groups for both women and men but more often in geriatric patients. This is due to physiological changes and co-morbidities that occur with age. Therefore, we decided to conduct one simulation experiment focusing exclusively on the ICD-G category. The aim of the experiment was to determine the necessary number of beds in neurological wards operating in WR hospitals to cover future demand. We assumed that the average length of stay in the ward will not change from what was recorded in 2018 , and that the required bed occupancy will be 0.85 (acceptable level) or 0.8 (optimal level). The simulation experiment was performed in two variants. The baseline scenario was compared with the scenario in which admission rates for older cohorts gradually increase starting from 2021 (Fig. 9). The growth of demand for neurological beds will put substantial pressure on the regional healthcare system, and 7 to 12 beds will need to be secured to cover the increased demand.

Fig. 9 Forecasted number of neurological beds. Legend: Scenario 1 (Sc1) is the baseline scenario; Scenario 4 (Sc4) considers increased admission rates for older cohorts, starting from 2021; the rates 0.8 and 0.85 comply with the optimal and acceptable bed occupancy rates

\section{Discussion}

In this study, we constructed a simulation model using the DES approach to investigate the impact of demographic changes on hospital demand in one administrative region in Poland, WR. We formulated health forecasts for different age-gender cohorts and various disease categories. We estimated that between 2018 and 2030, according to the baseline demographic scenario, the population of WR will increase by 3.79 , and this will result in a 4.8 increase in the total number of patients. Due to the continuing predominance of women in the overall population of WR, the demand generated by the female population will increase by 5.1 , while the number of male patients will grow by 4.2 .

A simulation of incidence in individual disease groups shows non-linear increases in cases. Fig.10 presents the percentage increases in incidence in subsequent years in five selected categories, separately for women and men. There are upward trends in all categories of diseases, but the dynamics and intensity of the trends vary. Very high growth rates are projected for ICD-D1 (neoplasms) and ICD-E (metabolic diseases) in the male population and for ICD-E in the female population. Meanwhile, for the ICD-G (nervous system) disease group in male cohorts and ICD-G and ICD-I (circulatory system) in female cohorts, the dynamics of growth are rather flat.

Fig. 10 Forecasted percentage increase of incidence (with reference to 2018), separately for men (a) and women (b). Legend: C, D1, E, G, I - aggregated codes for ICD10 disease classification, see Table 4

Ageing will likely have an important impact on morbidity trends. Between 2018 and 2030, if nothing changes other than the age distribution of the population, the demand for hospital services generated only by the oldest cohorts (70+) will grow very dynamically and rapidly (Fig.11). This is particularly well-illustrated by the ICD-G (nervous system) disease category. Meanwhile, for the population as a whole, the percentage increase (calculated with reference to 2018 ) is positive but small and flat. For the age group 70+, the incremental values are observed to be rising sharply.

Fig. 11 Forecasted percentage increase of incidence (with reference to 2018) separately for men (a) and women (b); Legend: calculated only for cohorts 70+; C, D1, E, G, I - aggregated codes for ICD10 disease classification, see Table 4

Using a simulation experiment, we determined the number of beds in neurological wards that will meet the increased predicted needs of the WR population. This forecast also includes beds in geriatric wards, which are dedicated to patients aged $85+$ years. Studies have shown the need to increase the number of such beds each year, even if the current age-gender specific demand rates are maintained. However, it is more likely that these indicators will change towards increasing demand for health services. Such a scenario will further strengthen the trend resulting solely from population ageing.

\section{Conclusion}

The methodological approach presented in this paper can be a valuable instrument for regional healthcare planners, as it can help in flexibly adjusting the supply (e.g. number of beds) to the forecasted demand. The financial resources at the disposal of regional decision-makers should be allocated in a way that takes into account projected changes and that improves the efficiency of the hospital supply network. This type of research also raises the question of the impact of new technologies on the number of hospitalisations in older age groups-whether new technologies will lead to increased demand among older patients or on the contrary, will be much more strongly targeted at younger age groups. In such a case, analyses of projected quantitative changes in the whole population would be more appropriate than only in the oldest age group. 
This research exhibited the potential for using simulation modelling to gain better understanding of the impact of demographic phenomena on healthcare demand; however, it is not complete. We plan to expand the simulation model so that it is possible to formulate forecasts not only at the regional level but also taking into account the specificity and capabilities of individual hospitals and hospital wards.

\section{Abbreviations}

DES: discrete event simulation; SD: system dynamics; ABS: agent-based simulation, MSM: microsimulation; WR: Wrocław region; NHF: National Health Fund

\section{Declarations}

\section{Ethics approval and consent to participate}

Administrative approval for the use of patients' data from internal databases that were not publicly accessible was provided by National Health Fund. The patients' administrative data used in this study were anonymised at the individual level, and the patient's identity and other sensitive data were not disclosed. According to Polish Law no ethical approval is needed when data is anonymised in such a way that the data subject cannot be identified anymore. The study was performed in full compliance with Polish law on personal data protection: Journal of Laws 2018 item 1000 and Journal of Laws 2020 item 849 . No administrative permissions were required to access and use the demographic data described in the study.

\section{Funding}

This research was financed by a grant from the National Science Centre (NSC), Poland, titled Simulation modelling of the demand for healthcare services. It was awarded based on decision 2015/17/B/HS4/00306. The NSC has no involvement in any research activity beyond providing funding, and has no role in the study design, data collection, analyses, or interpretation.

\section{Availability of data and materials}

The patient data used in the study are from administrative health records of the Regional Health Brand and are not publicly available. Access to data was allowed exclusively for the scientific purposes of this grant and after full anonymisation of sensitive data. Demographic data are publicly available at the Central Statistical Office webpage (www.stat.gov.pl). The processed datasets used and analysed during the current study are available from the corresponding author upon reasonable request.

\section{Consent for publication}

Not applicable

\section{Competing interests}

The author declares that she has no competing interests.

\section{Acknowledgment}

Not applicable

\section{Author details}

Wrocław University of Science and Technology, wyb. Wyspiańskiego 27, 50-370 Wrocław, Department of Operational Research and Business Intelligence

\section{References}

1. Strunk BC, Ginsburg PB, Banker MI. The effect of population aging on future hospital demand. Health Aff. 2006;25(3):w149.

2. Burkett E, Martin-Khan MG, Scott J, Samanta M, Gray LC. Trends and predicted trends in presentations of older people to Australian emergency departments: effects of demand growth, population aging and climate change. Aust Heal Rev. 2017;41(3):246-53.

3. Aboagye-Sarfo P, Mai Q, Sanfilippo FM, Fatovich DM. Impact of population ageing on growing demand for emergency transportation to emergency departments in Western Australia, 2005-2020. Emerg Med Australas. 2016;28(5):551-7.

4. Rand K, Dahl FA, Viana J, Rønning OM, Faiz KW, Barra M. Fewer ischemic strokes, despite an ageing population: stroke models from observed incidence in Norway 2010-2015. BMC Health Serv Res. 2019;19(1):705.

5. Demir E, Southern D, Rashid S, Lebcir R. A discrete event simulation model to evaluate the treatment pathways of patients with cataract in the United Kingdom. BMC Health Serv Res. 2018;18(1):933.

6. Vrhovec J, Tajnikar M. Population ageing and healthcare demand: The case of Slovenia. Health Policy (New York). 2016;120(11):1329-36.

7. Astolfi R, Lorenzoni L, Oderkirk J. A comparative analysis of health forecasting methods. 2012;(59). https://www.oecdilibrary.org/content/paper/5k912j389bf0-en

8. Roberfroid D, Leonard C, Stordeur S. Physician supply forecast: better than peering in a crystal ball? Hum Resour Health. 2009;7:1-13.

9. Jalalpour M, Gel Y, Levin S. Forecasting demand for health services: Development of a publicly available toolbox. Oper Res Heal Care. 2015;5:1-9.

10. Soyiri IN, Reidpath DD. An overview of health forecasting. Environ Health Prev Med. 2013;18(1):1-9. 
11. OBrien Antognini JM, Antognini JF, Khatri V. How many operating rooms are needed to manage non-elective surgical cases? A Monte Carlo simulation study. BMC Health Serv Res. 2015;15:487.

12. Sugiyama T, Goryoda S, Inoue K, Sugiyama-Ihana N, Nishi N. Construction of a simulation model and evaluation of the effect of potential interventions on the incidence of diabetes and initiation of dialysis due to diabetic nephropathy in Japan. BMC Health Serv Res. 2017;17(1):833.

13. Alibrahim A, Wu S. An agent-based simulation model of patient choice of health care providers in accountable care organizations. Health Care Manag Sci. 2018;21(1):131-43.

14. Bae K-H, Jones M, Evans G, Antimisiaris D. Simulation modelling of patient flow and capacity planning for regional long-term care needs: a case study. Heal Syst. 2019;8(1):1-16.

15. Kingston A, Comas-Herrera A, Jagger C. Forecasting the care needs of the older population in England over the next 20 years: estimates from the Population Ageing and Care Simulation (PACSim) modelling study. Lancet Public Heal. 2018;3:e447-55.

16. Harper A, Mustafee N, Feeney M. A hybrid approach using forecasting and discrete-event simulation for endoscopy services. In: 2017 Winter Simulation Conference (WSC). 2017. p. 1583-94.

17. Kittipittayakorn C, Ying K-C. Using the integration of discrete event and agent-based simulation to enhance outpatient service quality in an orthopedic department. J Healthc Eng. 2016;2016:4189206.

18. Ansah JP, Korne D De, Bayer S, Pan C, Jayabaskar T, Matchar DB, et al. Future requirements for and supply of ophthalmologists for an aging population in Singapore. Hum Resour Health. 2015;13:86.

19. Senese F, Tubertini P, Mazzocchetti A, Lodi A, Ruozi C, Grilli R. Forecasting future needs and optimal allocation of medical residency positions: the EmiliaRomagna Region case study. Hum Resour Health. 2015;13(1):7.

20. Barber P, Lopez-Valcarcel B. Forecasting the need for medical specialists in Spain: application of a system dynamics model. Hum Resour Health. 2010;8(1):24.

21. Davis $P$, Lay-Yee R, Pearson J. Using micro-simulation to create a synthesised data set and test policy options: The case of health service effects under demographic ageing. Health Policy (New York). 2010;97(2-3):267-74.

22. Schofield DJ, Earnest A. Demographic change and the future demand for public hospital care in Australia, 2005 to 2050. Aust Heal Rev. 2006;30(4):50715.

23. Mielczarek B. Combining simulation techniques to understand demographic dynamics and forecast hospital demands. In: Proceedings of the Winter Simulation Conference. IEEE Press; 2019;1114-1125.

24. Mielczarek B, Zabawa J. Simulation model for studying impact of demographic, temporal, and geographic factors on hospital demand. In: Proceedings of the Winter Simulation Conference. Institute of Electrical and Electronics Engineers Inc.; 2017;4498-500.

25. Zhang X. Application of discrete event simulation in health care: a systematic review. BMC Health Serv Res. 2018;18(1):687.

26. Law AM, Kelton WD. Simulation modeling and analysis. New York: McGraw-Hill Higher Education; 2000.

27. Nentwich LM, Grimmnitz B. Neurologic emergencies in the elderly. Emergency Medicine Clinics of North America. 2016;34:575-99.

\section{Figures}




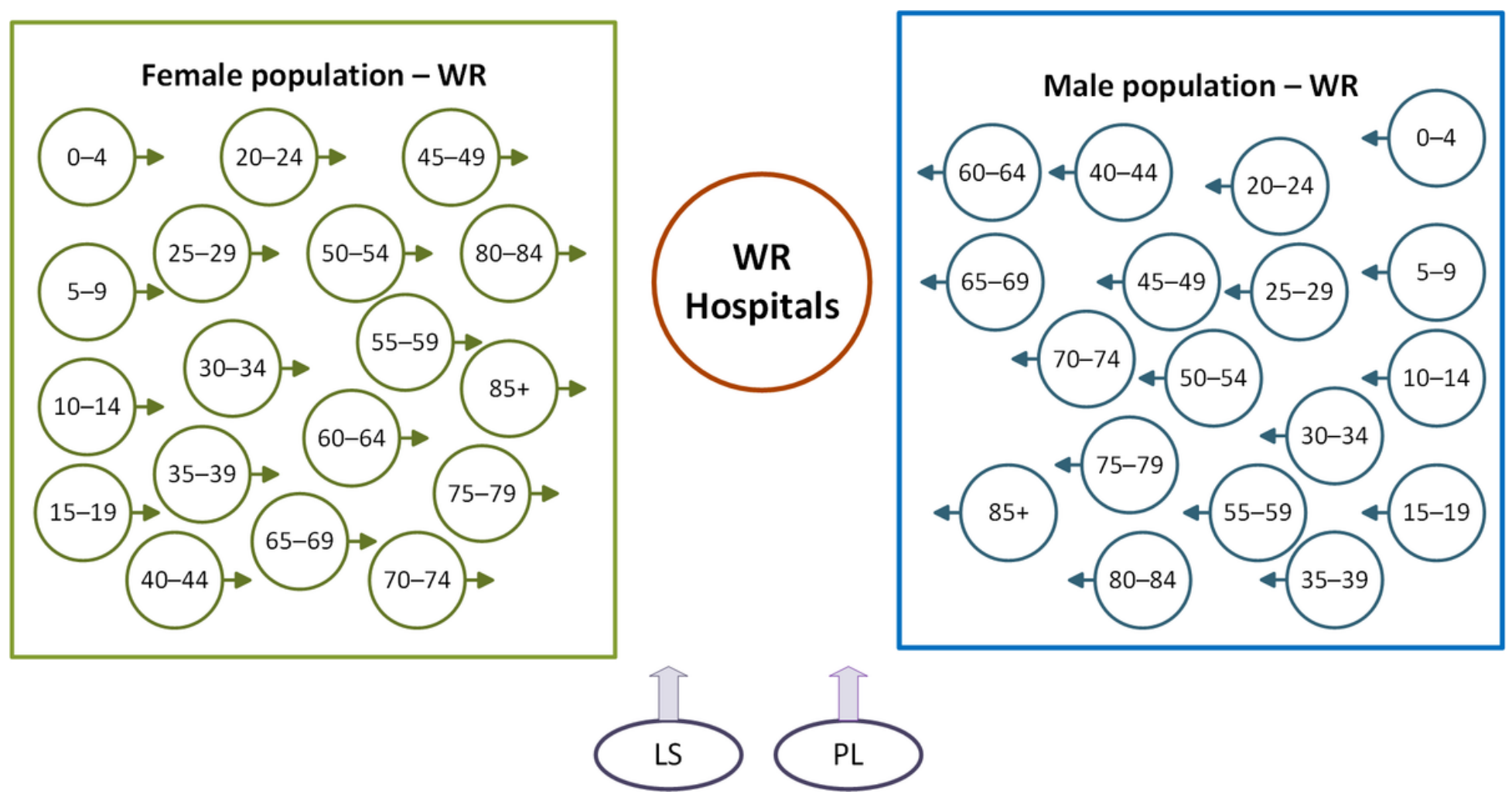

Figure 1

Patients' arrival at Wrocław region (WR) hospitals. Legend: Each age-gender cohort of WR residents corresponds to patients who arrive at WR hospitals. Additionally, patients from Lower Silesia (LS) and other regions of Poland (PL) may also register at the WR hospitals.

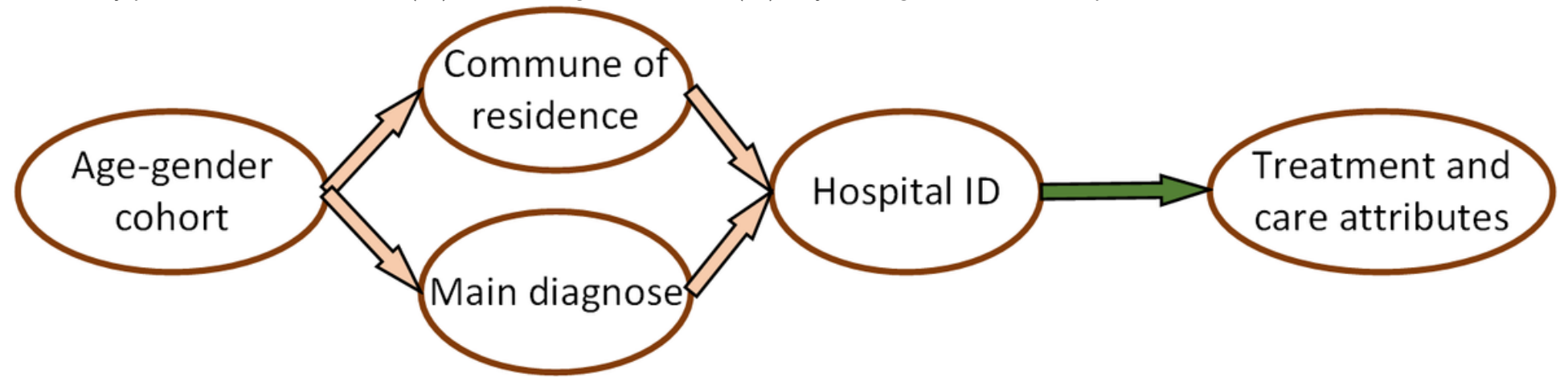

Figure 2

Relationships between patients' attributes in the discrete event simulation model 


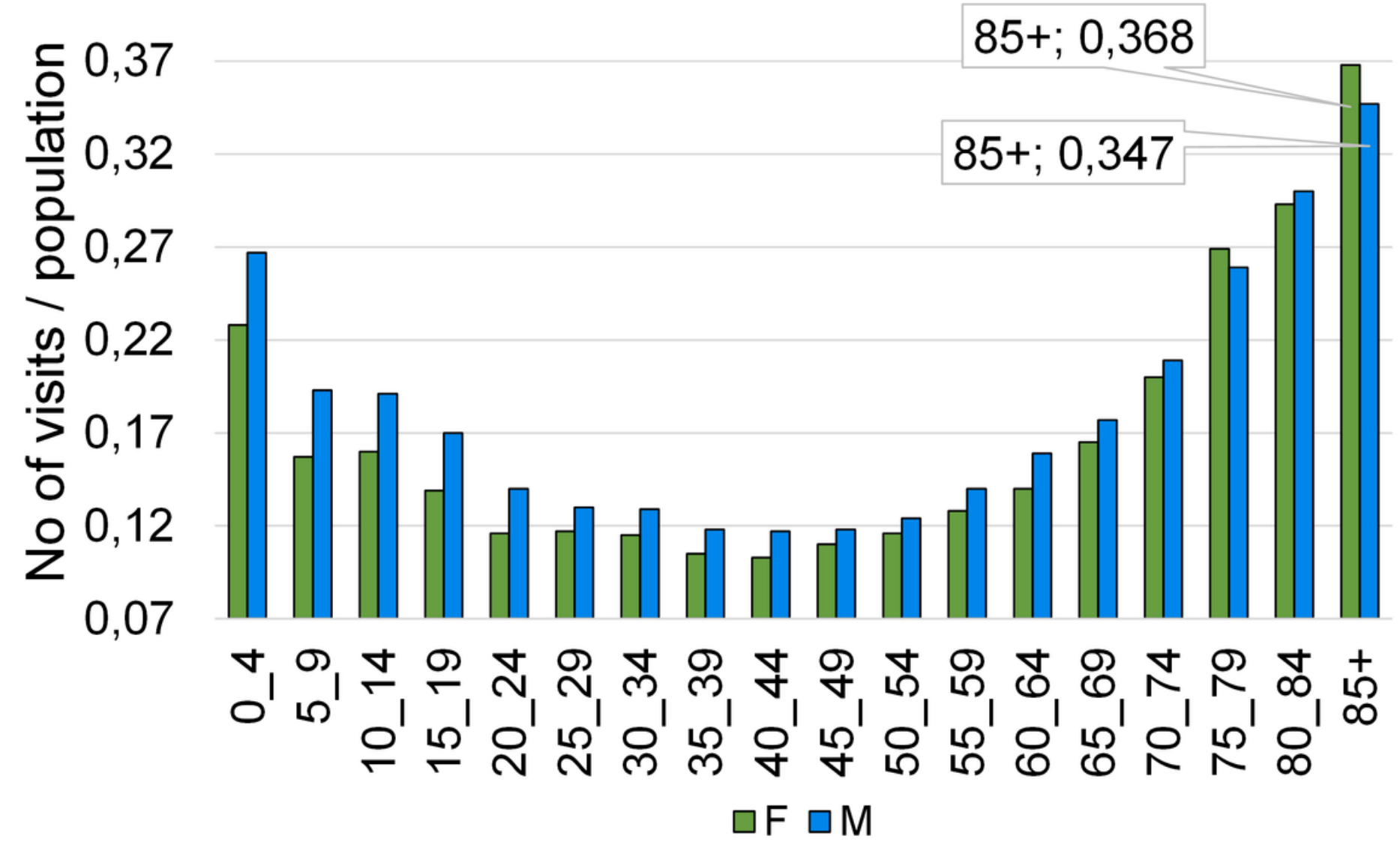

Figure 3

Age-gender specific demand rates for the Wrocław region population

a

Figure 4

Monthly trends of arrivals by gender. Legend: A comparison of historical (2011) and simulation data (average values from 10 replications) for (a) female and (b) male population We carried out a large number of tests to check the model's response to a sudden and rapid increase or decrease in demand. We also validated the model through visual demonstrations to NHF decision makers and healthcare professionals. After testing the model, we conclude that the model has a high degree of face validity. 


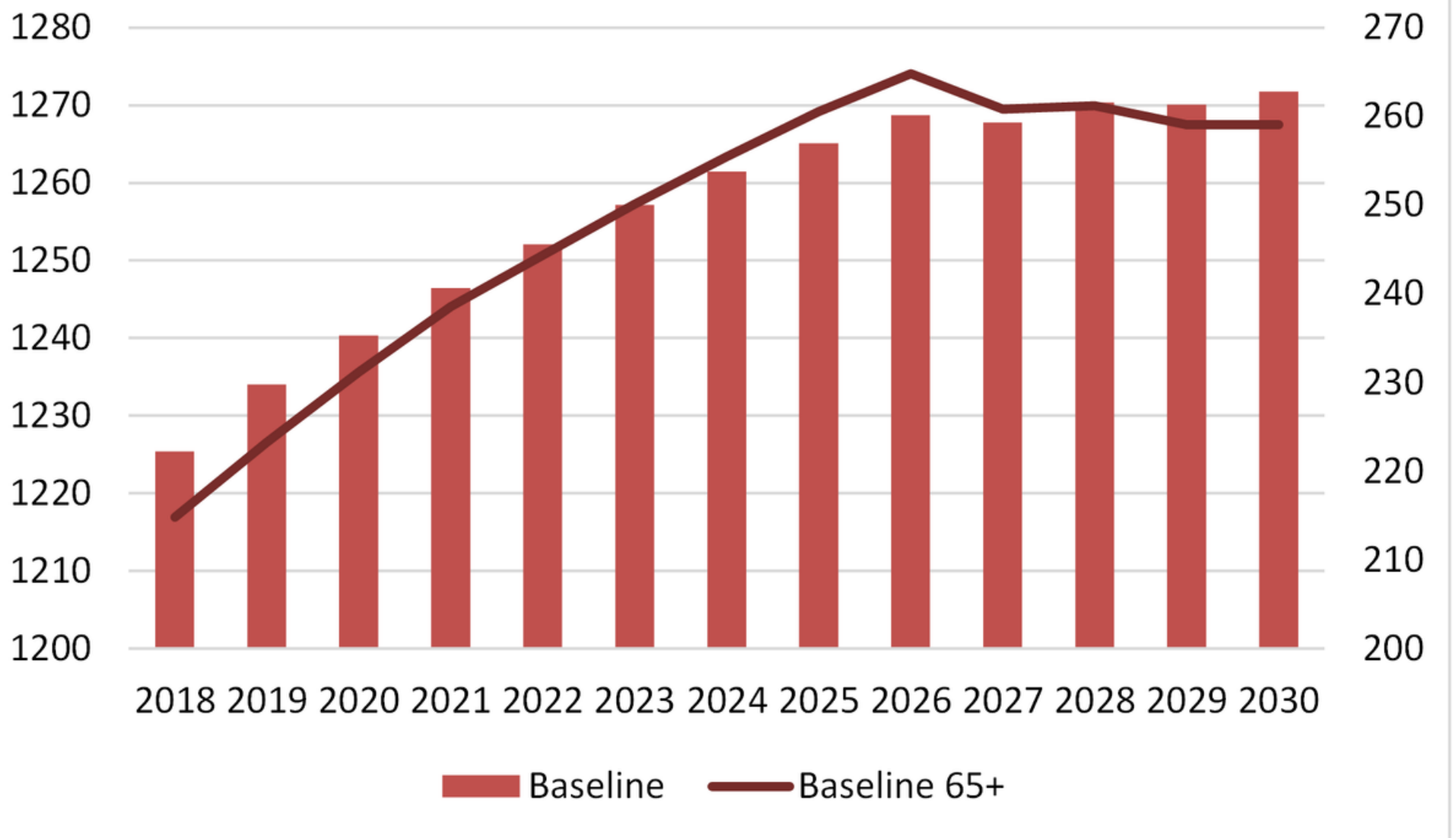

Figure 5

Demographic changes in the Wrocław region population according to the baseline scenario. Legend: the bar chart shows the projected population of the region from 2018 to 2030, and the solid line shows changes in the population aged 65+ years.
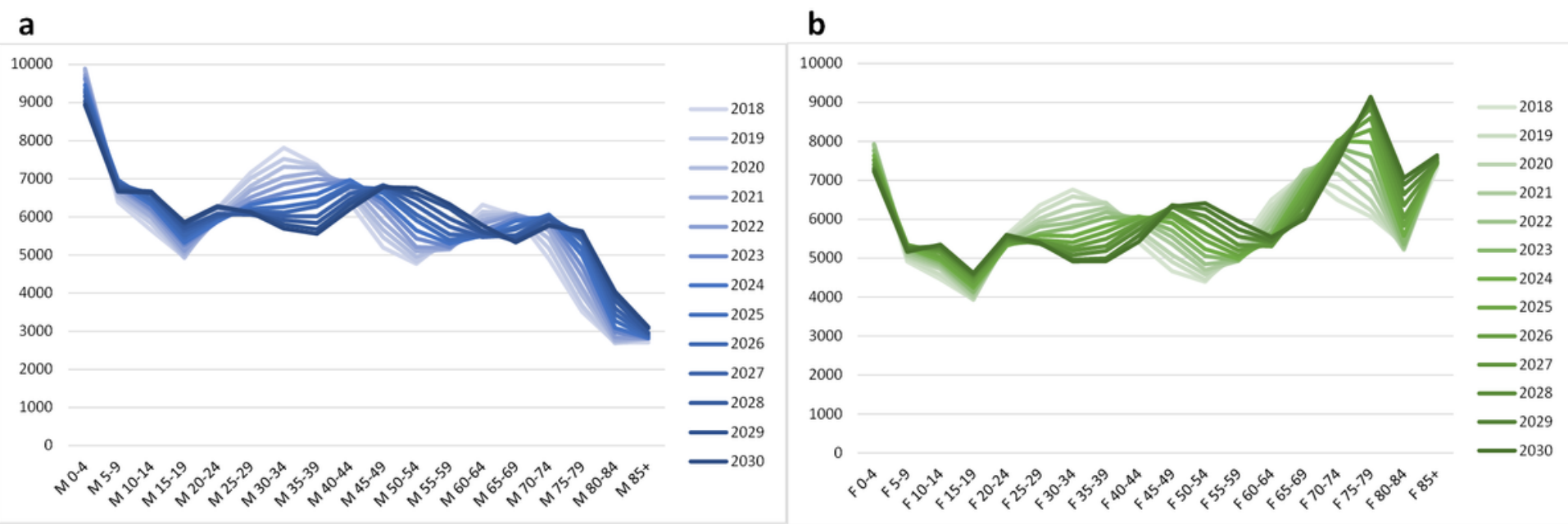

\section{Figure 6}

Total hospital demand according to the baseline scenario: Male population (left) and female population (right) 


\section{$80,0 \%$}

$60,0 \%$

$40,0 \%$

$20,0 \%$

$0,0 \%$

$-20,0 \%$

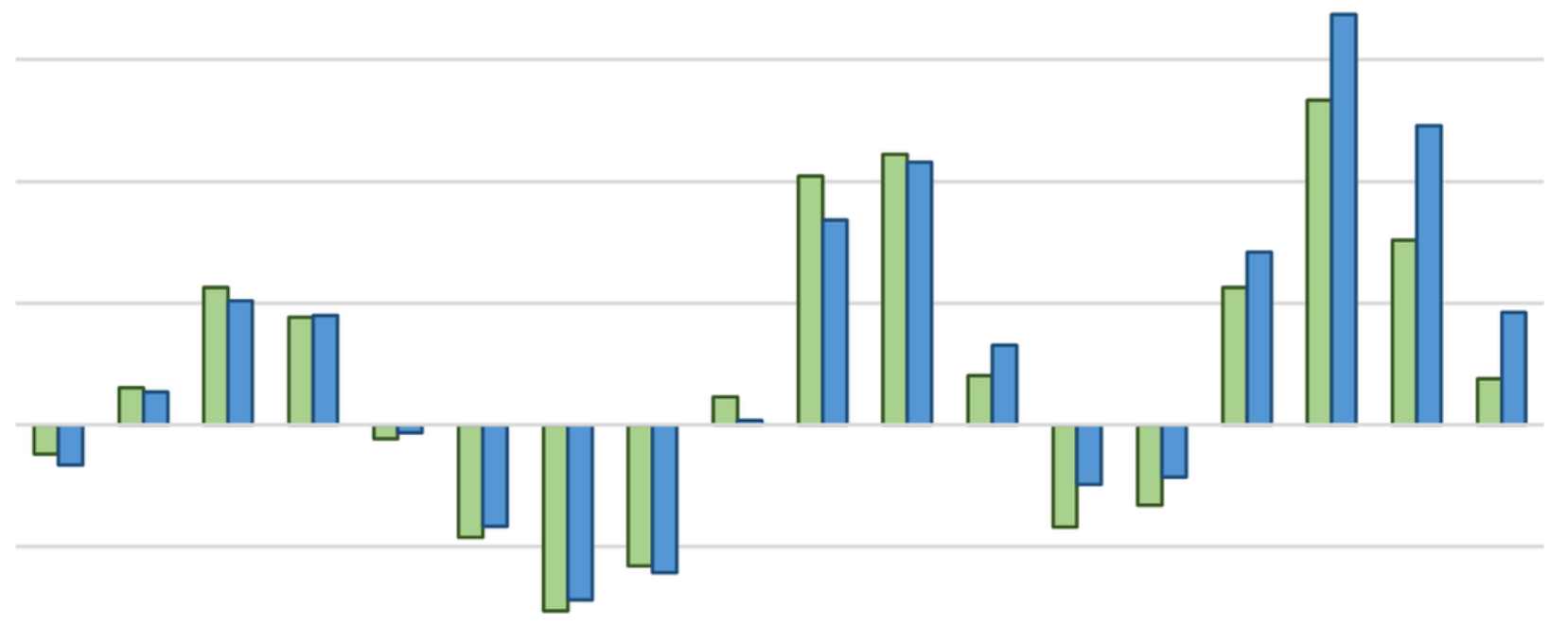

$-40,0 \%$

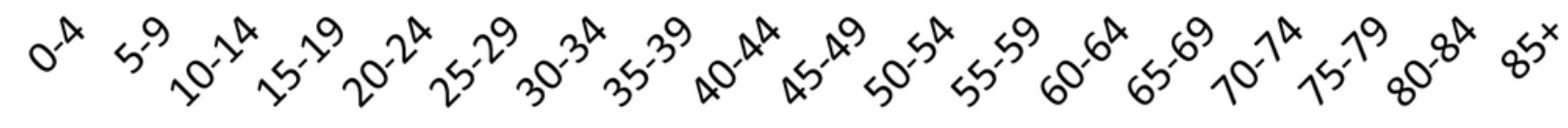

\section{Female $\square$ Male}

\section{Figure 7}

Percentage changes in the total hospital demand between 2018 and 2030 according to the baseline scenario 


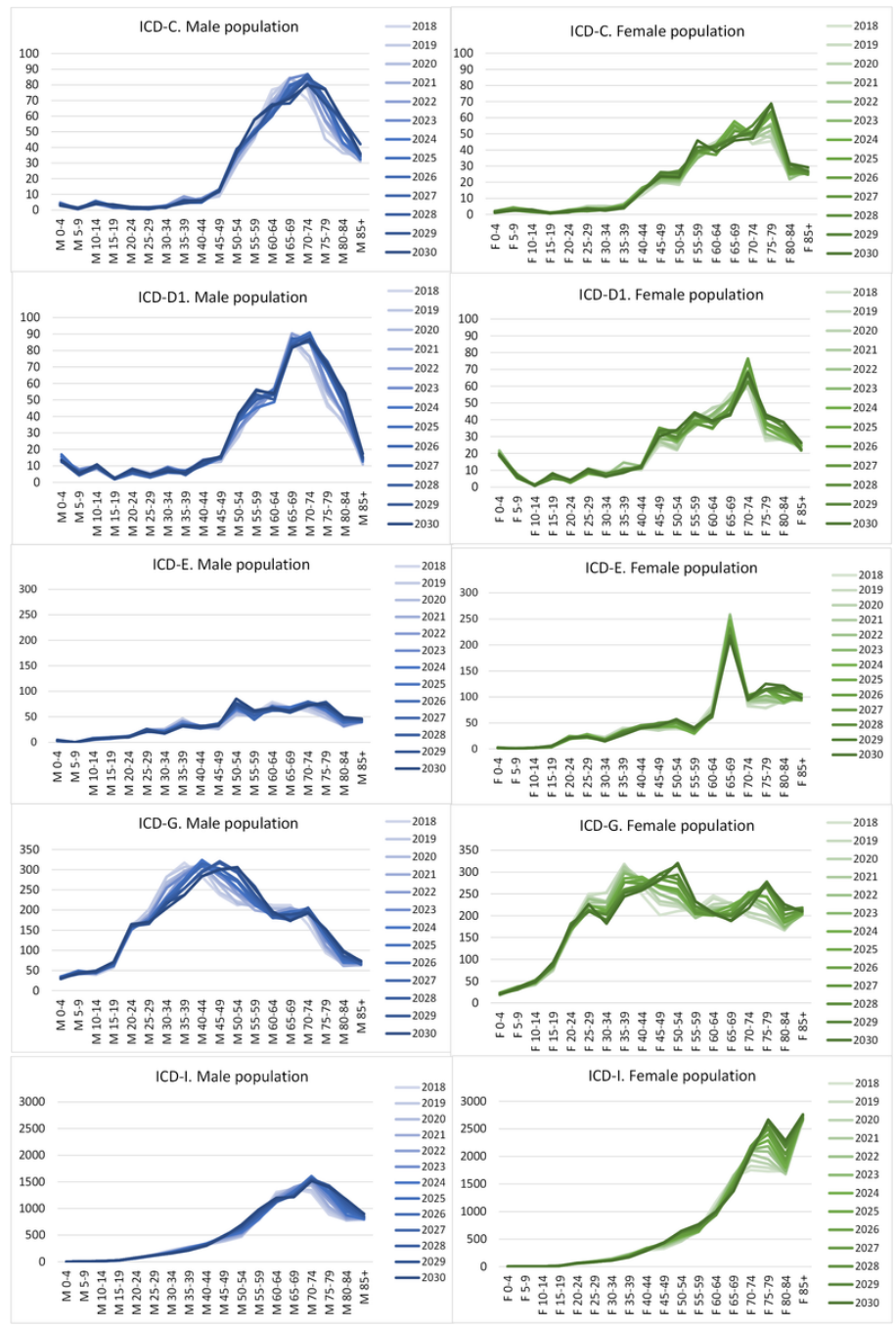

Figure 8

Forecasts of the incidence rates in selected disease groups. Legend: The graphs show those groups of diseases for which a significantly high share of patients aged over 70 years is observed 


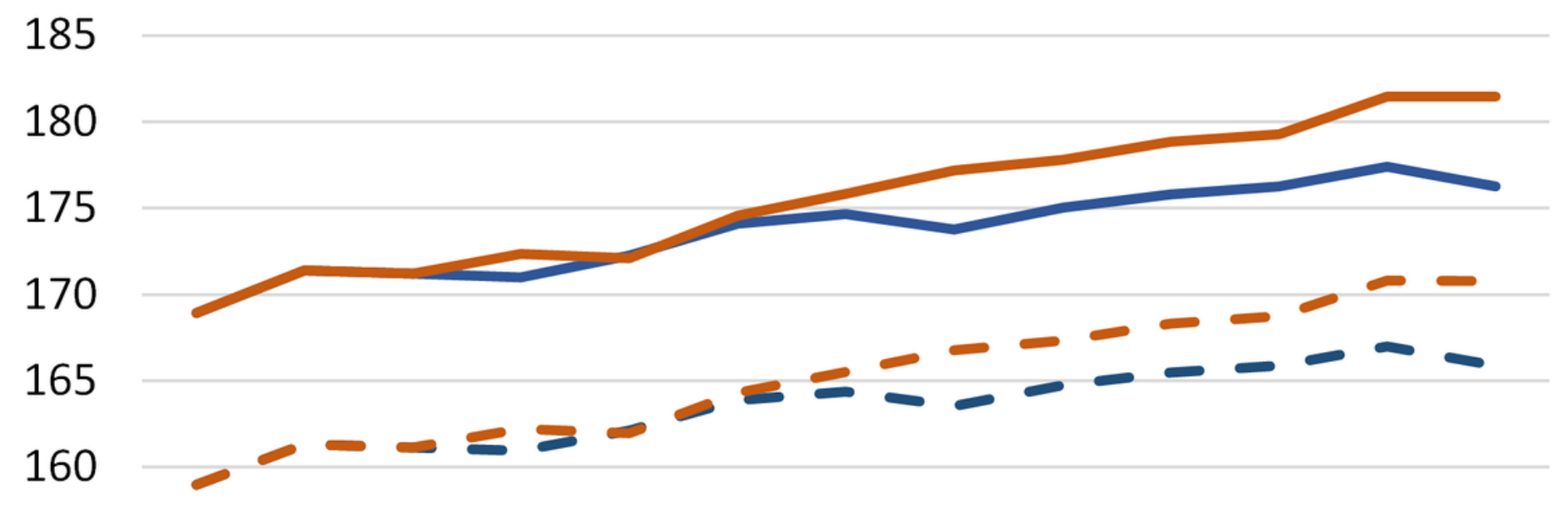

155

150

145

\section{0 $\longrightarrow$ Sc1_0.8 Sc4_0.8 - Sc1_0.85- - Sc4_0.85}

\section{Figure 9}

Forecasted number of neurological beds. Legend: Scenario 1 (Sc1) is the baseline scenario; Scenario 4 (Sc4) considers increased admission rates for older cohorts, starting from 2021; the rates 0.8 and 0.85 comply with the optimal and acceptable bed occupancy rates

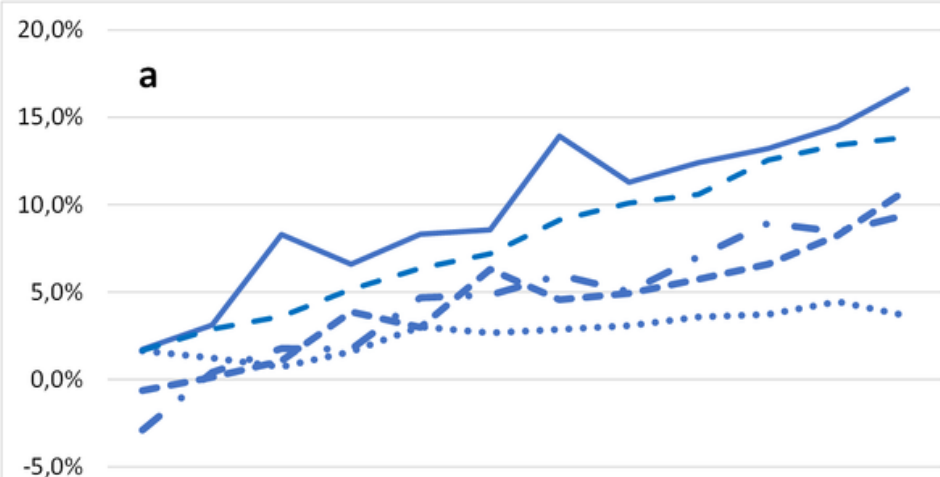

201920202021202220232024202520262027202820292030

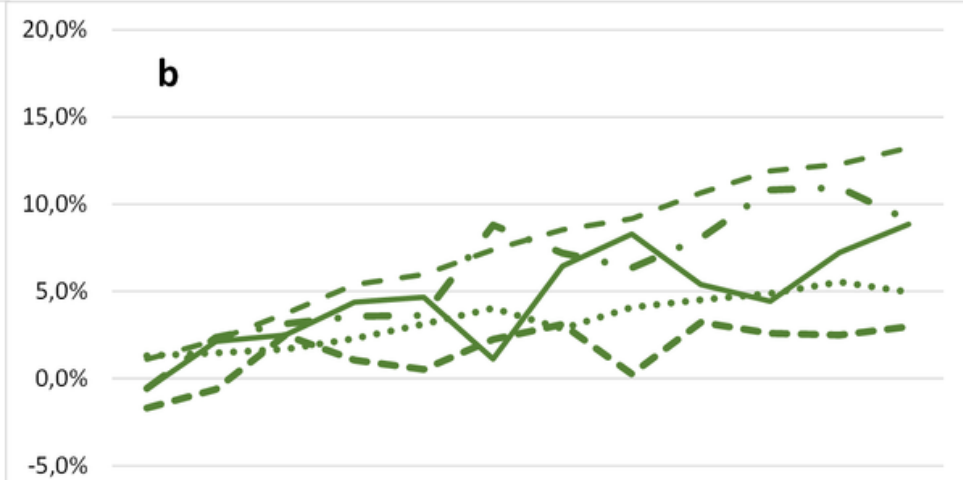

201920202021202220232024202520262027202820292030 $-\cdot \mathrm{C}-\mathrm{D} 1--\cdot \mathrm{E} \cdot \mathrm{G} \cdot \mathrm{G}--1$

Figure 10

Forecasted percentage increase of incidence (with reference to 2018), separately for men (a) and women (b). Legend: C, D1, E, G, I - aggregated codes for ICD10 disease classification, see Table 4 


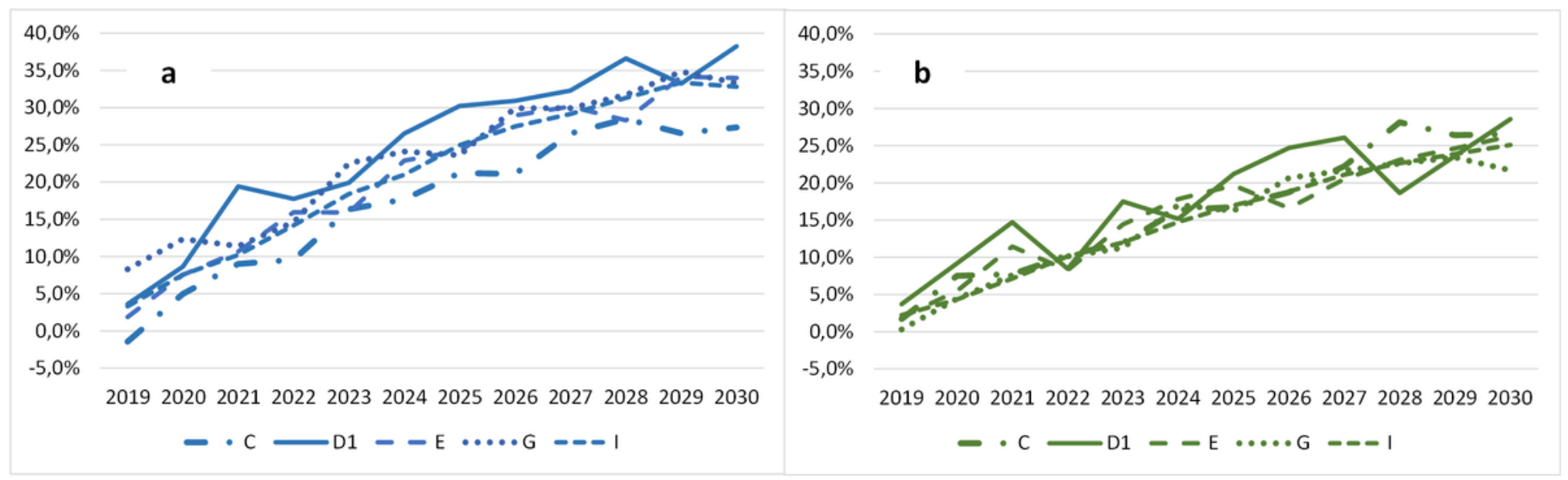

\section{Figure 11}

Forecasted percentage increase of incidence (with reference to 2018) separately for men (a) and women (b); Legend: calculated only for cohorts 70+; C, D1, E, G, I - aggregated codes for ICD10 disease classification, see Table 4 